\title{
$s$-Inversion Sequences and $P$-Partitions of Type $B$
}

\author{
William Y.C. Chen ${ }^{1}$, Alan J.X. Guo ${ }^{2}$, Peter L. Guo ${ }^{3}$ \\ Harry H.Y. Huang ${ }^{4}$, Thomas Y.H. Liu ${ }^{5}$ \\ ${ }^{1,2,3,4,5}$ Center for Combinatorics, LPMC-TJKLC \\ Nankai University \\ Tianjin 300071, P.R. China \\ ${ }^{1}$ chen@nankai.edu.cn, ${ }^{2}$ aalen@mail.nankai.edu.cn, ${ }^{3} \operatorname{lguo@nankai.edu.cn~}$ \\ ${ }^{4}$ hhuang@cfc.nankai.edu.cn, ${ }^{5}$ lyh@cfc.nankai.edu.cn
}

\begin{abstract}
Given a sequence $s=\left(s_{1}, s_{2}, \ldots\right)$ of positive integers, the inversion sequences with respect to $s$, or $s$-inversion sequences, were introduced by Savage and Schuster in their study of lecture hall polytopes. A sequence $\left(e_{1}, e_{2}, \ldots, e_{n}\right)$ of nonnegative integers is called an $s$-inversion sequence of length $n$ if $0 \leq e_{i}<s_{i}$ for $1 \leq i \leq n$. Let $I(n)$ be the set of $s$-inversion sequences of length $n$ for $s=(1,4,3,8,5,12, \ldots)$, that is, $s_{2 i}=4 i$ and $s_{2 i-1}=2 i-1$ for $i \geq 1$, and let $P_{n}$ be the set of signed permutations on $\left\{1^{2}, 2^{2}, \ldots, n^{2}\right\}$. Savage and Visontai conjectured that when $n=$ $2 k$, the ascent number over $I_{n}$ is equidistributed with the descent number over $P_{k}$. For a positive integer $n$, we use type $B P$-partitions to give a characterization of signed permutations over which the descent number is equidistributed with the ascent number over $I_{n}$. When $n$ is even, this confirms the conjecture of Savage and Visontai. Moreover, let $I_{n}^{\prime}$ be the set of $s$-inversion sequences of length $n$ for $s=(2,2,6,4,10,6, \ldots)$, that is, $s_{2 i}=2 i$ and $s_{2 i-1}=4 i-2$ for $i \geq 1$. We find a set of signed permutations over which the descent number is equidistributed with the ascent number over $I_{n}^{\prime}$.
\end{abstract}

Keywords: inversion sequence, signed permutation, type $B P$-partition, equidistribution

AMS Subject Classifications: 05A05, 05A15

\section{Introduction}

The notion of $s$-inversion sequences was introduced by Savage and Schuster [8] in their study of lecture hall polytopes. Let $s=\left(s_{1}, s_{2}, \ldots\right)$ be a sequence of positive integers. An inversion sequence of length $n$ with respect to $s$, or an $s$-inversion sequence of length $n$, is a sequence $e=\left(e_{1}, e_{2}, \ldots, e_{n}\right)$ of nonnegative integers such that $0 \leq e_{i}<s_{i}$ for 
$1 \leq i \leq n$. An ascent of an $s$-inversion sequence $e=\left(e_{1}, e_{2}, \ldots, e_{n}\right)$ is defined to be an integer $i \in\{0,1, \ldots, n-1\}$ such that

$$
\frac{e_{i}}{s_{i}}<\frac{e_{i+1}}{s_{i+1}}
$$

under the assumption that $e_{0}=0$ and $s_{0}=1$. The ascent number $\operatorname{asc}(e)$ of $e$ is meant to be the number of ascents of $e$.

The generating function of the ascent number over $s$-inversion sequences can be viewed as a generalization of the Eulerian polynomial for permutations, since the ascent number over the $s$-inversion sequences of length $n$ for $s=(1,2,3, \ldots)$ is equidistributed with the descent number over the permutations on $\{1,2, \ldots, n\}$, see Savage and Schuster [8]. For an inversion sequence $e=\left(e_{1}, e_{2}, \ldots, e_{n}\right)$ with respect to $s=\left(s_{1}, s_{2}, \ldots,\right)$, let

$$
\operatorname{amaj}(e)=\sum_{i \in \operatorname{Asc}(e)}(n-i)
$$

and

$$
\operatorname{lhp}(e)=-|e|+\sum_{i \in \operatorname{Asc}(e)}\left(s_{i+1}+\cdots+s_{n}\right)
$$

where $\operatorname{Asc}(e)$ is the set of ascents of $e$, and $|e|=e_{1}+e_{2}+\cdots+e_{n}$ is the weight of $e$. Savage and Schuster [8] showed that the multivariate generating function for the ascent number ase $(e)$, the major index amaj(e), the lecture hall statistic $\operatorname{lhp}(e)$ and the weight $|e|$ is related to the Ehrhart series of $s$-lecture hall polytopes and the generating function of $s$-lecture hall partitions.

Savage and Visontai [9] found a connection between the generating function of the ascent number over $s$-inversion sequences of length $n$ and a conjecture of Brenti [3. on the real-rootedness of Eulerian polynomials of finite Coxeter groups. The real-rootedness of the Eulerian polynomial of type $A$ was known to Frobenius [6], see also [2, 7]. Brenti [3] proved the real-rootedness of the Eularian polynomials of Coxeter groups of type $B$ and exceptional Coxeter groups. For the sequence $s=(2,4,6, \ldots)$ and an $s$-inversion sequence $e=\left(e_{1}, e_{2}, \ldots, e_{n}\right)$, Savage and Visontai [9] defined the type $D$ ascent set of $e$ as given by

$$
\operatorname{Asc}_{D}(e)=\left\{i \mid \frac{e_{i}}{i}<\frac{e_{i+1}}{i+1}, 1 \leq i \leq n-1\right\} \cup\left\{0 \mid \text { if } 2 e_{1}+e_{2} \geq 3\right\} .
$$

Let $T_{n}(x)$ be the generating function of the type $D$ ascent number over $s$-inversion sequence of length $n$ for $s=(2,4,6, \ldots)$. For example, $T_{3}(x)=2\left(x^{3}+11 x^{2}+11 x+1\right)$. Let $D_{n}(x)$ be the $n$-th Eulerian polynomial of type $D$. Recall that the type $D$ Coxeter group of rank $n$, denoted $D_{n}$, is the group of even-signed permutations on $\{1,2, \ldots, n\}$, see Björner and Brenti [1]. The descent set $\operatorname{Des}_{D}(\sigma)$ of an even-signed permutation $\sigma=\sigma_{1} \sigma_{2} \cdots \sigma_{n} \in D_{n}$ is defined by

$$
\operatorname{Des}_{D}(\sigma)=\left\{i \mid \sigma_{i}>\sigma_{i+1}, 1 \leq i \leq n-1\right\} \cup\left\{0 \mid \text { if } \sigma_{1}+\sigma_{2}<0\right\} .
$$


The descent number of $\sigma$ is meant to be the number of descents in $\operatorname{Des}_{D}(\sigma)$. Let $D_{n}(x)$ denote the generating function of the descent number over $D_{n}$. Savage and Visontai [9] showed that $T_{n}(x)=2 D_{n}(x)$. By proving that $T_{n}(x)$ has only real roots for $n \geq 1$, they deduced the real-rootedness of the Eulerian polynomials of type $D$ and settled the last unsolved case of the conjecture of Brenti [3].

Savage and Visontai [9] proved that for any sequence $s$ of positive integers and any positive integer $n$, the generating function of the ascent number over $s$-inversion sequences of length $n$ has only real roots. Let $I_{n}$ denote the set of $s$-inversion sequences of length $n$ for the specific sequence $s=(1,4,3,8,5,12, \ldots)$, that is, for $i \geq 1, s_{2 i}=4 i$ and $s_{2 i-1}=2 i-1$. Let $P_{n}$ denote the set of signed permutations on the multiset $\left\{1^{2}, 2^{2}, \ldots, n^{2}\right\}$. Savage and Visontai [9] posed the following conjecture, which implies the real-rootedness of the generating function of the descent number over $P_{n}$.

Conjecture 1.1 ([9, Conjecture 3.27]) For $n \geq 1$, the descent number over $P_{n}$ is equidistributed with the ascent number over $I_{2 n}$.

In this paper, we give a proof of Conjecture 1.1, Let $P_{n}(x)$ denote the generating function of the descent number over the set $P_{n}$ of signed permutations on $\left\{1^{2}, 2^{2}, \ldots, n^{2}\right\}$, and let $I_{n}(x)$ denote the generating function of the ascent number over $I_{n}$. Savage and Schuster [8] found a relation for $I_{n}(x)$. We show that the generating function $P_{n}(x)$ equals the generating function of the descent number over linear extensions of certain signed labeled forests. By using $P$-partitions of type $B$ introduced by Chow [5], we show that the generating function for the descent number over linear extensions satisfies the same relation as $I_{2 n}(x)$. Thus the generating function $P_{n}(x)$ satisfies the same relation as $I_{2 n}(x)$. This proves Conjecture 1.1.

We also find characterizations of signed permutations such that the descent number is equidistributed with the ascent number over three other classes of $s$-inversion sequences. To be specific, we show that the descent number over the set of signed permutations on the multiset $\left\{1^{2}, 2^{2}, \ldots,(n-1)^{2}, n\right\}$ such that $n$ is assigned a minus sign is equidistributed with the ascent number over $I_{2 n-1}$. For $s=(2,2,6,4,10,6, \ldots)$, that is, for $i \geq 1, s_{2 i}=2 i$ and $s_{2 i-1}=4 i-2$, let $I_{n}^{\prime}$ denote the set of $s$-inversion sequences of length $n$. We show that the descent number over $P_{n}$ is equidistributed with the ascent number over $I_{2 n}^{\prime}$ and the descent number over the set of signed permutations on $\left\{1^{2}, 2^{2}, \ldots,(n-1)^{2}, n\right\}$ is equidistributed with the ascent number over $I_{2 n-1}^{\prime}$.

\section{Proof of Conjecture 1.1}

In this section, we present a proof of Conjecture 1.1 by establishing a connection between the generating function $P_{n}(x)$ of the descent number over $P_{n}$ and the generating function of the descent number over linear extensions of certain signed labeled forests with $2 n$ 
vertices. Let $F_{n}$ be the plane forest with $n$ trees containing exactly two vertices, and let $F_{n}(x)$ denote the generating function of the descent number over linear extensions of $\left(F_{n}, w\right)$, where $w$ ranges over certain signed labelings of $F_{n}$. Keep in mind that a plane forest means a set of plane trees that are arranged in linear order. We shall show that $F_{n}(x)=P_{n}(x)$. On the other hand, by using $P$-partitions of type $B$ introduced by Chow [5], we obtain a relation for $F_{n}(x)$. Savage and Schuster [8] have shown that the same relation is satisfied by the generating function $I_{2 n}(x)$, so we get $I_{2 n}(x)=F_{n}(x)$. This confirms Conjecture 1.1, that is, $P_{n}(x)=I_{2 n}(x)$.

Let us give an overview of linear extensions of a signed labeled forest. Let $F$ be a plane forest with $n$ vertices, and let $S$ be a set of $n$ distinct positive integers. A labeling of $F$ on $S$ is an assignment of the elements in $S$ to the vertices of $F$ such that each element in $S$ is assigned to only one vertex. A signed labeling of $F$ on $S$ is a labeling of $F$ on $S$ with each label possibly associated with a minus sign. For example, Figure 2.1 illustrates a signed labeled forest on $\{1,2, \ldots, 9\}$. We use $(F, w)$ to stand for a plane
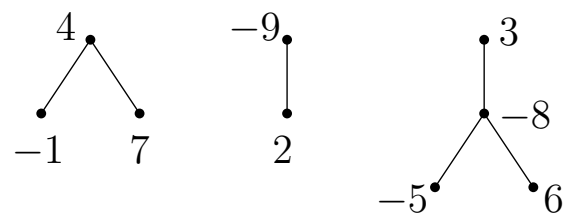

Figure 2.1: A signed labeled forest on $\{1,2, \ldots, 9\}$

forest $F$ associated with a signed labeling $w$.

Linear extensions of a signed labeled forest $(F, w)$ are defined based on linear extensions of $F$. For a plane forest $F$ with $n$ vertices, say $x_{1}, x_{2}, \ldots, x_{n}$, a linear extension of $F$ is a permutation $x_{i_{1}} x_{i_{2}} \cdots x_{i_{n}}$ of the vertices of $F$ such that $x_{i_{j}}<_{F} x_{i_{k}}$ implies $j<k$, where $<_{F}$ is the order relation of $F$. Let $\mathcal{L}(F)$ denote the set of linear extensions of $F$. Then the set of linear extensions of $(F, w)$ is defined as

$$
\mathcal{L}(F, w)=\left\{w\left(x_{i_{1}}\right) w\left(x_{i_{2}}\right) \cdots w\left(x_{i_{n}}\right) \mid x_{i_{1}} x_{i_{2}} \cdots x_{i_{n}} \in \mathcal{L}(F)\right\}
$$

where $w(x)$ denotes the label of a vertex $x$ of $F$.

Notice that a linear extension of $(F, w)$ is a signed permutation on the labeling set $S$ of $F$. We next define the generating function $F_{n}(x)$ of the descent number over linear extensions of the plane forest $F_{n}$ associated with certain signed labelings. Let us recall the descent number of a signed permutation on a multiset. A signed permutation on a multiset $M$ is a permutation on $M$ for which each element is possibly assigned a minus sign. For example, $\overline{3} 1 \overline{2} 13$ is a signed permutation on $\left\{1^{2}, 2,3^{2}\right\}$, where we use a bar to indicate that an element is assigned a minus sign. The descent set of a signed permutation $\sigma=\sigma_{1} \sigma_{2} \cdots \sigma_{n}$ is defined as

$$
\left\{i \mid \sigma_{i}>\sigma_{i+1}, 1 \leq i \leq n-1\right\} \cup\left\{0 \mid \text { if } \sigma_{1}<0\right\},
$$


see Savage and Visontai [9]. However, for the purpose of this paper, we use the following reformation of the descent set of a signed permutation $\sigma=\sigma_{1} \sigma_{2} \cdots \sigma_{n}$

$$
\operatorname{Des}_{B}(\sigma)=\left\{i \mid \sigma_{i}>\sigma_{i+1}, 1 \leq i \leq n-1\right\} \cup\left\{n \mid \text { if } \sigma_{n}>0\right\}
$$

The number of descents in $\operatorname{Des}_{B}(\sigma)$ is referred to as the descent number of $\sigma$, denoted $\operatorname{des}_{B}(\sigma)$. In fact, via the bijection

$$
\sigma=\sigma_{1} \sigma_{2} \cdots \sigma_{n} \longmapsto \sigma^{\prime}=\left(-\sigma_{n}\right)\left(-\sigma_{n-1}\right) \cdots\left(-\sigma_{1}\right),
$$

we see that the descent numbers defined by (2.1) and $\operatorname{Des}_{B}(\sigma)$ are equidistributed over signed permutations on any multiset.

To define the generating function $F_{n}(x)$, we introduce some specific singed labelings for the plane forest $F_{n}$. We write $T_{1}, T_{2}, \ldots, T_{n}$ for the $n$ trees of $F_{n}$, which are listed from left to right. For $1 \leq i \leq n$, let $u_{i}$ denote the root of $T_{i}$ and let $v_{i}$ denote the child of $u_{i}$. Let $w$ be a signed labeling of $F_{n}$ on $\{1,2, \ldots, 2 n\}$ such that for $1 \leq i \leq n$,

$$
\left\{\left|w\left(u_{i}\right)\right|,\left|w\left(v_{i}\right)\right|\right\}=\{2 i-1,2 i\} .
$$

Let $w_{i}(1 \leq i \leq n)$ be the signed labeling of $T_{i}$ induced by $w$. There are eight possibilities for each $w_{i}$. However, for the purpose of establishing the following equidistribution theorem, we need only four cases as given below:

Case 1: $w_{i}\left(u_{i}\right)=2 i$ and $w_{i}\left(v_{i}\right)=2 i-1$;

Case 2: $w_{i}\left(u_{i}\right)=2 i$ and $w_{i}\left(v_{i}\right)=\overline{2 i-1}$;

Case 3: $w_{i}\left(u_{i}\right)=\overline{2 i}$ and $w_{i}\left(v_{i}\right)=2 i-1$;

Case 4: $w_{i}\left(u_{i}\right)=\overline{2 i-1}$ and $w_{i}\left(v_{i}\right)=\overline{2 i}$.

For the $j$-th case, we say that $w_{i}$ is of type $j$. Let $L\left(F_{n}\right)$ denote the set of signed labelings of $F_{n}$ such that the induced labeling $w_{i}$ of $T_{i}$ is one of the above four types. We shall show that the descent number over $P_{n}$ is equidistributed with the descent number over the set of linear extensions of $\left(F_{n}, w\right)$, where $w$ ranges over the set $L\left(F_{n}\right)$. Specifically, define

$$
F_{n}(x)=\sum_{w \in L\left(F_{n}\right)} \sum_{\sigma \in \mathcal{L}\left(F_{n}, w\right)} x^{\operatorname{des}_{B}(\sigma)}
$$

We have the following equidistribution theorem.

Theorem 2.1 For $n \geq 1$, we have

$$
F_{n}(x)=P_{n}(x)
$$


Proof. We proceed to construct a descent preserving bijection $\phi$ from the set

$$
\left\{\sigma \in \mathcal{L}\left(F_{n}, w\right) \mid w \in L\left(F_{n}\right)\right\}
$$

to the set $P_{n}$ of signed permutations on $\left\{1^{2}, 2^{2}, \ldots, n^{2}\right\}$. Let $\sigma=\sigma_{1} \sigma_{2} \cdots \sigma_{2 n}$ be a linear extension in $\mathcal{L}\left(F_{n}, w\right)$, where $w \in L\left(F_{n}\right)$. Define $\phi(\sigma)=\tau=\tau_{1} \tau_{2} \cdots \tau_{2 n}$ as follows. For $1 \leq j \leq 2 n, \tau_{j}$ has the same sign as $\sigma_{j}$, and $\left|\tau_{j}\right|=i$ if $\left|\sigma_{j}\right|=2 i-1$ or $\left|\sigma_{j}\right|=2 i$. It is routine to check that $\phi$ is a bijection. Moreover, it is readily verified that $j \in\{1,2, \ldots, 2 n\}$ is a descent of $\sigma$ if and only if it is a descent of $\tau$. This completes the proof.

The next theorem gives an expression for the generating function $F_{n}(x)$.

Theorem 2.2 For $n \geq 1$, we have

$$
\frac{F_{n}(x)}{(1-x)^{2 n+1}}=\sum_{t \geq 0}(t+1)^{n}(2 t+1)^{n} x^{t} .
$$

To prove Theorem 2.2, we need a decomposition of $P$-partitions of type $B$ into $\sigma$ compatible maps due to Chow [5], where $\sigma$ is a linear extension of $P$. When the poset $P$ is associated with an ordinary labeling, type $B P$-partitions reduce to ordinary $P$ partitions introduced by Stanley [10]. To make a connection to Theorem 2.2, it is sufficient to consider the case when $P$ is a plane forest. In this case, we do not need the structure of $P$-partitions of type $B$ in full generality as given by Chow. For the case when $P$ is a plane forest, a $P$-partition of type $B$ was described by Chen, Gao and Guo [4].

Let $F$ be a plane forest, and $w$ be a signed labeling of $F$. Let $\mathbb{N}$ be the set of nonnegative integers. A $(F, w)$-partition of type $B$ is a map $f$ from the set of vertices of $F$ to $\mathbb{N}$ that satisfies the following conditions:

(1) $f(x) \leq f(y)$ if $x \geq_{F} y$;

(2) $f(x)<f(y)$ if $x>_{F} y$ and $w(x)<w(y)$;

(3) $f(x) \geq 1$ if $x$ is a root of $F$ with $w(x)>0$.

Analogous to the decomposition of ordinary $P$-partitions given by Stanley [10], Chow [5] showed that type $B(F, w)$-partitions can be decomposed into $\sigma$-compatible maps, where $\sigma$ is a linear extension of $(F, w)$. For a signed permutation $\sigma=\sigma_{1} \sigma_{2} \cdots \sigma_{n}$, a $\sigma$-compatible map $g$ is a map from $\left\{\sigma_{1}, \sigma_{2}, \ldots, \sigma_{n}\right\}$ to $\mathbb{N}$ that satisfies the following conditions:

(1) $g\left(\sigma_{1}\right) \geq g\left(\sigma_{2}\right) \geq \cdots \geq g\left(\sigma_{n}\right)$;

(2) For $i \in\{1,2, \ldots, n-1\}, g\left(\sigma_{i}\right)>g\left(\sigma_{i+1}\right)$ if $\sigma_{i}>\sigma_{i+1}$; 
(3) $g\left(\sigma_{n}\right) \geq 1$ if $\sigma_{n}>0$

Let $A(F, w)$ denote the set of type $B(F, w)$-partitions, and let $A_{\sigma}$ denote the set of $\sigma$-compatible maps. The following decomposition is due to Chow [5].

Theorem 2.3 ([5, Theorem 2.1.4]) Let $F$ be a plane forest associated with a signed labeling $w$. Then

$$
A(F, w)=\bigcup_{\sigma \in \mathcal{L}(F, w)} A_{\sigma} .
$$

For a nonnegative integer $t$, let $\Omega_{F}(w, t)$ denote the number of type $B(F, w)$ partitions $f$ such that $f(x) \leq t$ for any $x \in F$. When $w$ is an ordinary labeling, Stanley [10] has established a relation between the generating function of the descent number over linear extensions of $(F, w)$ and the generating function of $\Omega_{F}(w, t)$. For signed labeled forests, we have the following relation.

Theorem 2.4 Let $F$ be a plane forest with $n$ vertices, and $w$ be a signed labeling of $F$ on $\{1,2, \ldots, n\}$. Then

$$
\frac{\sum_{\sigma \in \mathcal{L}(F, w)} x^{\operatorname{des}_{B}(\sigma)}}{(1-x)^{n+1}}=\sum_{t \geq 0} \Omega_{F}(w, t) x^{t} .
$$

Proof. We essentially follow the proof of Stanley [10] for ordinary $P$-partitions. For a signed permutation $\sigma=\sigma_{1} \sigma_{2} \cdots \sigma_{n}$ on $\{1,2, \ldots, n\}$, let $\Omega_{\sigma}(t)$ denote the number of $\sigma$-compatible maps $g$ with $g\left(\sigma_{1}\right) \leq t$. For any linear extension $\sigma$ in $\mathcal{L}(F, w)$, in view of the decomposition (2.4), relation (2.5) can be deduced from the following relation

$$
\sum_{t \geq 0} \Omega_{\sigma}(t) x^{t}=\frac{x^{\operatorname{des}_{B}(\sigma)}}{(1-x)^{n+1}} .
$$

For $1 \leq i \leq n$, let $d_{i}$ denote the number of descents of $\sigma$ that are greater than or equal to $i$, that is,

$$
d_{i}=\mid\left\{j \mid \sigma_{j} \geq \sigma_{j+1}, i \leq j \leq n-1\right\} \cup\left\{n \mid \text { if } \sigma_{n}>0\right\} \mid .
$$

Setting $\lambda_{i}=g\left(\sigma_{i}\right)-d_{i}$, we are led to a one-to-one correspondence between the set of $\sigma$-compatible maps $g$ with $g\left(\sigma_{1}\right) \leq t$ and the set of partitions $\left(\lambda_{1}, \lambda_{2}, \ldots, \lambda_{n}\right)$ with $\lambda_{1} \leq t-\operatorname{des}_{B}(\sigma)$, where the latter is counted by

$$
\left(\begin{array}{c}
n+t-\operatorname{des}_{B}(\sigma) \\
n
\end{array}\right)
$$


see, for example, Stanley [10]. Thus,

$$
\begin{aligned}
\sum_{t \geq 0} \Omega_{\sigma}(t) x^{t} & =\sum_{t \geq 0}\left(\begin{array}{c}
n+t-\operatorname{des}_{B}(\sigma) \\
n
\end{array}\right) x^{t} \\
& =\frac{x^{\operatorname{des}_{B}(\sigma)}}{(1-x)^{n+1}}
\end{aligned}
$$

which agrees with (2.6). This completes the proof.

We are now ready to prove Theorem 2.2 .

Proof of Theorem 2.2. By Theorem 2.4, the assertion (2.3) is equivalent to the following relation

$$
\sum_{w \in L\left(F_{n}\right)} \Omega_{F_{n}}(w, t)=((t+1)(2 t+1))^{n} .
$$

Let $T_{1}, T_{2}, \ldots, T_{n}$ be the $n$ trees of $F_{n}$ listed from left to right. Thus, for any signed labeled forest $\left(F_{n}, w\right)$, we have

$$
\Omega_{F_{n}}(w, t)=\prod_{i=1}^{n} \Omega_{T_{i}}\left(w_{i}, t\right),
$$

where $w_{i}$ is the signed labeling of $T_{i}$ induced by $w$. Recall that for a signed labeling $w$ in $L\left(F_{n}\right)$, each induced labeling $w_{i}$ has four choices. For $1 \leq j \leq 4$, let $w_{i}^{(j)}$ be the signed labeling of $T_{i}$ that is of type $j$, so that the left-hand side of (2.7) can be rewritten as

$$
\sum_{w \in L\left(F_{n}\right)} \Omega_{F_{n}}(w, t)=\prod_{i=1}^{n}\left(\Omega_{T_{i}}\left(w_{i}^{(1)}, t\right)+\Omega_{T_{i}}\left(w_{i}^{(2)}, t\right)+\Omega_{T_{i}}\left(w_{i}^{(3)}, t\right)+\Omega_{T_{i}}\left(w_{i}^{(4)}, t\right)\right) .
$$

We claim that for any $1 \leq i \leq n$,

$$
\Omega_{T_{i}}\left(w_{i}^{(1)}, t\right)+\Omega_{T_{i}}\left(w_{i}^{(2)}, t\right)+\Omega_{T_{i}}\left(w_{i}^{(3)}, t\right)+\Omega_{T_{i}}\left(w_{i}^{(4)}, t\right)=(t+1)(2 t+1) .
$$

Assume that $u_{i}$ is the root of $T_{i}$ and $v_{i}$ is the child of $u_{i}$. Let $f$ be a type $B\left(T_{i}, w_{i}^{(j)}\right)$ partition such that $f\left(v_{i}\right) \leq t$. Then we have

$$
\begin{array}{ll}
0<f\left(u_{i}\right) \leq f\left(v_{i}\right) \leq t, & \text { if } j=1 ; \\
0<f\left(u_{i}\right) \leq f\left(v_{i}\right) \leq t, & \text { if } j=2 ; \\
0 \leq f\left(u_{i}\right)<f\left(v_{i}\right) \leq t, & \text { if } j=3 \\
0 \leq f\left(u_{i}\right) \leq f\left(v_{i}\right) \leq t, & \text { if } j=4 .
\end{array}
$$

It follows that

$$
\Omega_{T_{i}}\left(w_{i}^{(1)}, t\right)=\Omega_{T_{i}}\left(w_{i}^{(2)}, t\right)=\Omega_{T_{i}}\left(w_{i}^{(3)}, t\right)=\left(\begin{array}{c}
t+1 \\
2
\end{array}\right)
$$


and

$$
\Omega_{T_{i}}\left(w_{i}^{(4)}, t\right)=\left(\begin{array}{c}
t+2 \\
2
\end{array}\right) .
$$

Hence we obtain (2.10), completing the proof.

In addition to Theorem 2.1 and Theorem 2.2, a formula of Savage and Schuster [8] is needed to prove Conjecture 1.1. Recall that $I_{n}$ is the set of $s$-inversion sequences of length $n$ for the specific sequence $s=(1,4,3,8,5,12, \ldots)$, and $I_{n}(x)$ is the generating function of the ascent number over $I_{n}$. Savage and Schuster [8, Theorem 13] showed that for $n \geq 1$,

$$
\frac{I_{n}(x)}{(1-x)^{n+1}}=\sum_{t \geq 0}(t+1)^{\left\lceil\frac{n}{2}\right\rceil}(2 t+1)^{\left\lfloor\frac{n}{2}\right\rfloor} x^{t} .
$$

Replacing $n$ by $2 n$ in (2.13), we get

$$
\frac{I_{2 n}(x)}{(1-x)^{2 n+1}}=\sum_{t \geq 0}(t+1)^{n}(2 t+1)^{n} x^{t} .
$$

Comparing (2.14) with (2.3), we obtain $F_{n}(x)=I_{2 n}(x)$. By Theorem 2.1 we arrive at $P_{n}(x)=I_{2 n}(x)$, completing the proof of Conjecture 1.1.

\section{$3 \quad$ Further equidistributions}

In this section, we give characterizations of three sets of signed permutations over which the descent number is equidistributed with the ascent number over the sets $I_{2 n-1}, I_{2 n-1}^{\prime}$ and $I_{2 n}^{\prime}$ respectively.

Recall that $I_{2 n-1}$ stands for the set of $s$-inversion sequences of length $2 n-1$ for $s=(1,4,3,8,5,12, \ldots)$, and $I_{n}^{\prime}$ stands for the set of $s$-inversion sequences of length $n$ for $s=(2,2,6,4,10,6, \ldots)$. Let $U_{n}$ be the set of signed permutations on the multiset $\left\{1^{2}, 2^{2}, \ldots,(n-1)^{2}, n\right\}$, and let $V_{n}$ be the subset of $U_{n}$ consisting of signed permutations such that the element $n$ carries a minus sign. We show that the descent number over $V_{n}$ is equidistributed with the ascent number over $I_{2 n-1}$, the descent number over $U_{n}$ is

equidistributed with the ascent number over $I_{2 n-1}^{\prime}$, and the descent number over $P_{n}$ is equidistributed with the ascent number over $I_{2 n}^{\prime}$.

Theorem 3.1 For $n \geq 1$, we have

$$
\sum_{\sigma \in V_{n}} x^{\operatorname{des}_{B}(\sigma)}=\sum_{e \in I_{2 n-1}} x^{\operatorname{asc}(e)} .
$$

Proof. Let $V_{n}(x)$ denote the sum on the left-hand side of (3.1). Since a relation on the generating function $I_{2 n-1}(x)$ is given by (2.13) , it suffices to show that $V_{n}(x)$ satisfies the 
same relation as $I_{2 n-1}(x)$, that is, for $n \geq 1$,

$$
\frac{V_{n}(x)}{(1-x)^{2 n}}=\sum_{t \geq 0}(t+1)^{n}(2 t+1)^{n-1} x^{t} .
$$

We claim that $V_{n}(x)$ equals the generating function of the descent number over linear extensions of certain signed labeled forests. To this end, let $F_{n}^{\prime}$ be the plane forest which is obtained from $F_{n-1}$ by adding a single vertex as the rightmost component. Let $T_{1}, T_{2}, \ldots, T_{n-1}$ denote $n-1$ trees of $F_{n-1}$, and let $T_{n}$ denote a single vertex. Hence $F_{n}^{\prime}$ consists of plane trees $T_{1}, \ldots, T_{n-1}, T_{n}$. Write $L\left(F_{n}^{\prime}\right)$ for the set of signed labelings $w$ of $F_{n}^{\prime}$ such that $w\left(T_{n}\right)=-(2 n-1)$ and the induced signed labeling of $w$ on $F_{n-1}$ belongs to $L\left(F_{n-1}\right)$. Set

$$
F_{n}^{\prime}(x)=\sum_{w \in L\left(F_{n}^{\prime}\right)} \sum_{\sigma \in \mathcal{L}\left(F_{n}^{\prime}, w\right)} x^{\operatorname{des}_{B}(\sigma)} .
$$

Using the same reasoning as in the proof of Theorem 2.1, one can construct a descent preserving bijection between the set

$$
\left\{\sigma \in \mathcal{L}\left(F_{n}^{\prime}, w\right) \mid w \in L\left(F_{n}^{\prime}\right)\right\}
$$

and the set $V_{n}$. Hence we get

$$
F_{n}^{\prime}(x)=V_{n}(x)
$$

so that (3.2) can be rewritten as

$$
\frac{F_{n}^{\prime}(x)}{(1-x)^{2 n}}=\sum_{t \geq 0}(t+1)^{n}(2 t+1)^{n-1} x^{t} .
$$

Applying Theorem 2.4 to the set of signed labeled forests $\left(F_{n}^{\prime}, w\right)$ with $w \in L\left(F_{n}^{\prime}\right)$, we obtain

$$
\frac{F_{n}^{\prime}(x)}{(1-x)^{2 n}}=\sum_{t \geq 0} \sum_{w \in L\left(F_{n}^{\prime}\right)} \Omega_{F_{n}^{\prime}}(w, t) x^{t} .
$$

Thus, (3.5) can be deduced from the following relation

$$
\sum_{w \in L\left(F_{n}^{\prime}\right)} \Omega_{F_{n}^{\prime}}(w, t)=(t+1)^{n}(2 t+1)^{n-1}
$$

Notice that for a signed labeling $w$ in $L\left(F_{n}^{\prime}\right)$, each induced labeling $w_{i}$ of $T_{i}$ for $1 \leq$ $i \leq n-1$ has four types, and the induced labeling $w_{n}$ of $T_{n}$ satisfies $w_{n}\left(T_{n}\right)=-(2 n-1)$. For $1 \leq i \leq n-1$ and $1 \leq j \leq 4$, let $w_{i}^{(j)}$ be the signed labeling of $T_{i}$ that is of type $j$. In the proof of Theorem 2.2 , it has been shown that for $1 \leq i \leq n-1$,

$$
\Omega_{T_{i}}\left(w_{i}^{(1)}, t\right)+\Omega_{T_{i}}\left(w_{i}^{(2)}, t\right)+\Omega_{T_{i}}\left(w_{i}^{(3)}, t\right)+\Omega_{T_{i}}\left(w_{i}^{(4)}, t\right)=(t+1)(2 t+1) .
$$


Moreover, it is clear that $\Omega_{T_{n}}\left(w_{n}, t\right)=t+1$. Hence we deduce that

$$
\begin{aligned}
\sum_{w \in L\left(F_{n}^{\prime}\right)} \Omega_{F_{n}^{\prime}}(w, t) & =\Omega_{T_{n}}\left(w_{n}, t\right) \prod_{i=1}^{n-1}\left(\Omega_{T_{i}}\left(w_{i}^{(1)}, t\right)+\Omega_{T_{i}}\left(w_{i}^{(2)}, t\right)+\Omega_{T_{i}}\left(w_{i}^{(3)}, t\right)+\Omega_{T_{i}}\left(w_{i}^{(4)}, t\right)\right) \\
& =(t+1)^{n}(2 t+1)^{n-1},
\end{aligned}
$$

as required.

We now show that the descent number over $P_{n}$ and $U_{n}$ is equidistributed with the ascent number over $I_{2 n}^{\prime}$ and $I_{2 n-1}^{\prime}$, respectively.

Theorem 3.2 For $n \geq 1$, we have

$$
\sum_{\sigma \in P_{n}} x^{\operatorname{des}_{B}(\sigma)}=\sum_{e \in I_{2 n}^{\prime}} x^{\operatorname{asc}(e)}
$$

and

$$
\sum_{\sigma \in U_{n}} x^{\operatorname{des}_{B}(\sigma)}=\sum_{e \in I_{2 n-1}^{\prime}} x^{\operatorname{asc}(e)}
$$

To prove Theorem 3.2, we need the following formulas (3.11) and (3.12) of Savage and Schuster [8] for the generating function of the ascent number over $s$-inversion sequences of length $n$. For a sequence $s=\left(s_{1}, s_{2}, \ldots\right)$ of positive integers, let $f_{n}^{(s)}(t)$ denote the number of sequences $\left(\lambda_{1}, \lambda_{2}, \ldots, \lambda_{n}\right)$ of nonnegative integers such that

$$
0 \leq \frac{\lambda_{1}}{s_{1}} \leq \frac{\lambda_{2}}{s_{2}} \leq \cdots \leq \frac{\lambda_{n}}{s_{n}} \leq t
$$

Theorem 3.3 ([즈, Theorem 5]) Let $s=\left(s_{1}, s_{2}, \ldots\right)$ be a sequence of positive integers. Then

$$
\sum_{t \geq 0} f_{n}^{(s)}(t) x^{t}=\frac{1}{(1-x)^{n+1}} \sum_{e} x^{\operatorname{asc}(e)},
$$

where e ranges over s-inversion sequences of length $n$.

In particular, for $s=(2,2,6,4,10,6, \ldots)$ and

$$
s^{\prime}=s / 2=(1,1,3,2,5,3, \ldots),
$$

Savage and Schuster [8, Theorem 14] showed that

$$
f_{n}^{\left(s^{\prime}\right)}(t)=(t+1)^{\left\lceil\frac{n}{2}\right\rceil}\left(\frac{t+2}{2}\right)^{\left\lfloor\frac{n}{2}\right\rfloor} .
$$

We now turn to the proof of Theorem 3.2. 
Proof of Theorem 3.2. It follows from (3.12) that

$$
f_{n}^{(s)}(t)=f_{n}^{\left(s^{\prime}\right)}(2 t)=(t+1)^{\left\lfloor\frac{n}{2}\right\rfloor}(2 t+1)^{\left\lceil\frac{n}{2}\right\rceil} .
$$

By Theorem 3.3, we obtain that

$$
\frac{\sum_{e \in I_{n}^{\prime}} x^{\operatorname{asc}(e)}}{(1-x)^{n+1}}=\sum_{t \geq 0}(t+1)^{\left\lfloor\frac{n}{2}\right\rfloor}(2 t+1)^{\left\lceil\frac{n}{2}\right\rceil} x^{t} .
$$

To prove (3.8), we replace $n$ by $2 n$ in (3.13) to get

$$
\frac{\sum_{e \in I_{2 n}^{\prime}} x^{\operatorname{asc}(e)}}{(1-x)^{2 n+1}}=\sum_{t \geq 0}(t+1)^{n}(2 t+1)^{n} x^{t} .
$$

Combining the above relation and the formula (2.14) for $I_{2 n}(x)$, we obtain

$$
\sum_{e \in I_{2 n}^{\prime}} x^{\operatorname{asc}(e)}=I_{2 n}(x) .
$$

Thus (3.8) follows from the fact that $I_{2 n}(x)=P_{n}(x)$.

Next we prove (3.9). Replacing $n$ by $2 n-1$ in (3.13), we get

$$
\frac{\sum_{e \in I_{2 n-1}^{\prime}} x^{\operatorname{asc}(e)}}{(1-x)^{2 n}}=\sum_{t \geq 0}(t+1)^{n-1}(2 t+1)^{n} x^{t} .
$$

Hence (3.9) can be deduced from the following relation

$$
\frac{\sum_{\sigma \in U_{n}} x^{\operatorname{des}_{B}(\sigma)}}{(1-x)^{2 n}}=\sum_{t \geq 0}(t+1)^{n-1}(2 t+1)^{n} x^{t} .
$$

To prove (3.15), let

$$
U_{n}(x)=\sum_{\sigma \in U_{n}} x^{\operatorname{des}_{B}(\sigma)} .
$$

We claim that $U_{n}(x)$ coincides with the generating function of the descent number over linear extensions of certain signed labeled forests. In the notation $F_{n}^{\prime}, T_{1}, \ldots, T_{n}$ as defined in the proof of Theorem [3.1, we use $\bar{L}\left(F_{n}^{\prime}\right)$ to denote the set of signed labelings $w$ of $F_{n}^{\prime}$ such that $w\left(T_{n}\right)=2 n-1$ or $-(2 n-1)$ and the induced labeling of $w$ on $F_{n-1}$ belongs to $L\left(F_{n-1}\right)$. Let

$$
G_{n}(x)=\sum_{w \in \bar{L}\left(F_{n}^{\prime}\right)} \sum_{\sigma \in \mathcal{L}\left(F_{n}^{\prime}, w\right)} x^{\operatorname{des}_{B}(\sigma)} .
$$


Again, using the argument in the proof of Theorem 2.1, one can construct a descent preserving bijection between the set

$$
\left\{\sigma \in \mathcal{L}\left(F_{n}^{\prime}, w\right) \mid w \in \bar{L}\left(F_{n}^{\prime}\right)\right\}
$$

and the set $U_{n}$. It then follows that

$$
G_{n}(x)=U_{n}(x)
$$

Therefore, (3.15) is equivalent to

$$
\frac{G_{n}(x)}{(1-x)^{2 n}}=\sum_{t \geq 0}(t+1)^{n-1}(2 t+1)^{n} x^{t} .
$$

Applying Theorem 2.4 to the set of signed labeled forests $\left(F_{n}^{\prime}, w\right)$ with $w \in \bar{L}\left(F_{n}^{\prime}\right)$, we find that

$$
\frac{G_{n}(x)}{(1-x)^{2 n}}=\sum_{t \geq 0} \sum_{w \in \bar{L}\left(F_{n}^{\prime}\right)} \Omega_{F_{n}^{\prime}}(w, t) x^{t} .
$$

Hence (3.17) can be deduced from the following relation

$$
\sum_{w \in \bar{L}\left(F_{n}^{\prime}\right)} \Omega_{F_{n}^{\prime}}(w, t)=(t+1)^{n-1}(2 t+1)^{n} .
$$

To prove (3.18), for $1 \leq i \leq n-1$ and $1 \leq j \leq 4$, let $w_{i}^{(j)}$ be the signed labeling of $T_{i}$ that is of type $j$, and let $w_{n}^{\prime}$ and $w_{n}^{\prime \prime}$ be the signed labelings of $T_{n}$ such that $w_{n}^{\prime}\left(T_{n}\right)=2 n-1$ and $w_{n}^{\prime \prime}\left(T_{n}\right)=-(2 n-1)$. As shown in the proof of Theorem 2.2 , for $1 \leq i \leq n-1$,

$$
\Omega_{T_{i}}\left(w_{i}^{(1)}, t\right)+\Omega_{T_{i}}\left(w_{i}^{(2)}, t\right)+\Omega_{T_{i}}\left(w_{i}^{(3)}, t\right)+\Omega_{T_{i}}\left(w_{i}^{(4)}, t\right)=(t+1)(2 t+1) .
$$

Evidently, $\Omega_{T_{n}}\left(w_{n}^{\prime}, t\right)=t$ and $\Omega_{T_{n}}\left(w_{n}^{\prime \prime}, t\right)=t+1$. Hence the sum on the left-hand side of (3.18) equals

$$
\begin{aligned}
& \left(\Omega_{T_{n}}\left(w_{n}^{\prime}, t\right)+\Omega_{T_{n}}\left(w_{n}^{\prime \prime}, t\right)\right) \prod_{i=1}^{n-1}\left(\Omega_{T_{i}}\left(w_{i}^{(1)}, t\right)+\Omega_{T_{i}}\left(w_{i}^{(2)}, t\right)+\Omega_{T_{i}}\left(w_{i}^{(3)}, t\right)+\Omega_{T_{i}}\left(w_{i}^{(4)}, t\right)\right) \\
& \quad=(t+1)^{n-1}(2 t+1)^{n}
\end{aligned}
$$

as required.

Acknowledgments. This work was supported by the 973 Project and the National Science Foundation of China. 


\section{References}

[1] A. Björner and F. Brenti, Combinatorics of Coxeter Groups, Gran. Texts in Math., vol. 231, Springer-Verlag, Berlin, 2005.

[2] M. Bóna, Combinatorics of Permutations, Discrete Mathematics and its Applications (Boca Raton), Chapman \& Hall/CRC, Boca Raton, FL, 2004.

[3] F. Brenti, $q$-Eulerian polynomials arising from Coxeter groups, European J. Combin. 15 (1994), 417-441.

[4] W.Y.C. Chen, O.X.Q. Gao and P.L. Guo, $q$-Hook length formulas for signed labeled forests, Adv. Appl. Math., to appear.

[5] C.-O. Chow, Noncommutative symmetric functions of type $B$, Ph.D. Thesis, MIT, 2001.

[6] G. Frobenius, Über die Bernoulli'schen Zahlen und die Euler'schen Polynome, Sitzungberichte der Königlich Preussischen Akademie der Wissenschaften (1910), Zweiter Halbband.

[7] J. Haglund and M. Visontai, Stable multivariate Eulerian polynomials and generalized Stirling permutations, European J. Combin. 33 (2012), 477-487.

[8] C.D. Savage and M.J. Schuster, Ehrhart series of lecture hall polytopes and Eulerian polynomials for inversion sequences, J. Combin. Theory Ser. A 119 (2012), 850-870.

[9] C.D. Savage and M. Visontai, The $s$-Eulerian polynomials have only real roots, Trans. Amer. Math. Soc., to appear.

[10] R.P. Stanley, Enumerative Combinatorics, Vol. 1, Cambridge University Press, Cambridge, 1997. 